\title{
Percepções de estudantes de medicina sobre a experiência de aprendizado na comunidade dentro do programa mais médicos: análise de um grupo focal.
}

\section{Medical student's perceptions of community-based learning experience in a primary care medical program: a focus-group analysis.}

\section{Percepciones de estudiantes de medicina en la experiencia de aprendizaje en la comunidad en el programa más médicos: análisis de un grupo de enfoque.}

\author{
Iuri Silva SENA ${ }^{1}$ \\ Lucas Coelho GUERREIRO ${ }^{1}$ \\ Atie Calado RIBEIRO ${ }^{1}$ \\ Leila do Socorro da SILVA MORAIS ${ }^{2}$ \\ Maira Tityomi Sacata Tongu NAZIMA ${ }^{3}$ \\ Bráulio Érison França dos $\mathrm{SANTO}^{4}$
}

RESUMO: O ensino médico orientado para a comunidade constitui um dos fundamentos do preparo de futuros profissionais para o trabalho nos serviços de saúde e permite formação ampla para atendimento nos diversos níveis de cuidado. A instituição do programa mais médicos permitiu distribuição de profissionais em regiões com maior necessidade e mudanças nos processos de trabalhos na atenção básica. O curso de medicina da Universidade Federal do Amapá (UNIFAP) iniciou em 2015 atividades de inserção de alunos nas equipes de saúde com profissionais do programa. Este estudo visou identificar as percepções dos acadêmicos de medicina sobre a experiência de aprendizado na comunidade durante acompanhamento de equipes de saúde da família do programa mais médicos. Realizou-se estudo qualitativo através da técnica do grupo

1 Acadêmico de Medicina da Universidade Federal do Amapá.

2 Professora Auxiliar de Medicina. Especialista em Pneumologia. Membro do Núcleo Docente Estruturante da Universidade Federal do Amapá.

3 Professora Adjunta de Medicina. Doutora em Oftalmologia. Coordenadora do Curso de Medicina da Universiade Federal do Amapá.

4 Professor Assistente de Medicina. Mestre em Ciências da Saúde. Membro do Núcleo Docente Estruturante da Universidade Federal do Amapá. 
focal com voluntários da quarta série de graduação de medicina. O estudo constatou que alunos tiveram percepções positivas sobre a atuação dos médicos acompanhados, principalmente pela valorização da relação médico paciente e atendimento holístico. Entretanto, houve identificação de necessidade de atualização dos protocolos brasileiros de atendimento na atenção primária. Os discentes mudaram visão preconcebida sobre a baixa qualidade profissional do programa e passaram a aceitar melhor a experiência. A observação da realidade estrutural de postos de saúde foi considerada causa importante para que médicos evitem trabalhar no interior ou regiões isoladas. Os achados auxiliam no aprofundamento das discussões sobre políticas de provimento de médicos e sua interação com o ensino, ainda assim novos estudos precisam ser estimulados para avaliar outros aspectos e impactos ocasionados por esse tipo de programa.

Palavras-chave: Médicos Graduados Estrangeiros, Grupos Focais, Medicina Comunitária

ABSTRACT: Community-based learning is one of the foundations of preparing future professionals to work in health services and allows extensive training to act in various levels of health care. In Brazil, The establishment of the "Mais Médicos" Program allowed distribution of professionals in areas with greater need and changes in primary care work processes. In 2015, the course of medicine at the Federal University of Amapá (UNIFAP) started students insertion activities in health teams with professionals of the Program. This study aimed to identify the perceptions of medical students on the learning experience in the community while following-up family health teams of the Program. A qualitative research was conducted through focus-group technique with fourth graders volunteers from medical school. Students had positive perceptions about the performance of doctors, mainly reagarding the doctor-patient relationship and holistic care. However, it was identified the need to update primary care to brazilian protocols. Students had their preconceived view of the low professional quality of the program changed and began to best accept the experience. The observation of the structural reality of health centers was considered an important cause to make doctors avoid working in the coutryside or isolated regions. These findings help to deepen the discussions on medical provision policies and their interaction with teaching. Neverthless, further studies need to be encouraged to evaluate other aspects and impacts caused by this type of program.

Keywords: Foreign Medical Graduates, Focus Groups, Community Medicine

RESUMEN: La educación médica orientada a comunidad es uno del fundamentos de preparación de futuros profesionales que trabajan en servicios de salud y permite una amplia formación para satisfacer diversos niveles de atención. El establecimiento del Programa Más Médicos permitido profesionales de distribución en zonas con mayor necesidad y cambios en procesos de trabajo en atención primaria. El curso de medicina en Universidad Federal de Amapá (UNIFAP) inició en 2015 actividades de inserción estudiantes en equipos de salud con profesionales del programa. Este estudio tuvo como objetivo identificar percepciones de estudiantes de medicina en la experiencia de aprendizaje en comunidad durante el seguimiento de equipos de salud de familia de programa más médico. Se realizó una investigación cualitativa mediante técnica de grupos focales con 


\section{$83 / /$}

los voluntarios de la cuarta serie de graduación médica. El estudio encontró que los estudiantes tenían percepciones positivas sobre el desempeño de médicos acompañados principalmente por apreciación de relación médico paciente y servicio integral. Sin embargo, se reconoció la necesidad de actualizar los protocolos de brasileños de atención en atención primaria. Los estudiantes cambiaron visión preconcebida de baja calidad profesional del programa y comenzaron a aceptar la mejor experiencia. La observación de realidad estructural de centros de salud se consideró importante causa de que médicos evitan trabajar dentro o regiones aisladas. Los resultados ayudan a profundizar las discusiones sobre provisión médica de políticas y su interacción con la enseñanza aún, más estudios necesitan ser alentados a evaluar otros aspectos e impactos causados por este tipo de programas.

Palabras clave: Médicos Graduados Extranjeros, Grupos Focales, Medicina Comunitaria

\section{INTRODUÇÃO}

A atualização das diretrizes curriculares nacionais (DCN) para cursos de graduação em Medicina, a partir da resolução $n^{\circ} 03$ de 20/06/2014, veio reforçar uma formação médica voltada para os diferentes níveis de atenção à saúde, com ênfase na atenção primária e na formação profissional que possa estabelecer uma relação humana com o paciente, pautados na responsabilidade social e na defesa da cidadania e da dignidade humana ${ }^{1}$.

O graduando em medicina deve ter oportunidade, ao longo do curso, de compreender as três vertentes que englobam o ensino médico. A primeira está relacionada com a atenção em saúde, a qual abrange dimensões referentes aos aspectos legislativos e éticos do Sistema Único de Saúde (SUS), como o estudo e aplicação dos conceitos de universalidade, equidade e integralidade. A segunda está voltada para a gestão em saúde, na qual o médico deverá ter ciência das políticas que envolvem o sistema de saúde e como se dá o seu funcionamento. A terceira envolve a educação em saúde, segundo a qual o médico deve ser promotor de sua própria educação continuada, além de agir como disseminador de conteúdo atualizado no meio em que atua ${ }^{1}$.

O curso de medicina da Universidade Federal do Amapá - UNIFAP já surgiu com estrutura curricular organizada na metodologia pedagógica da Aprendizagem Baseada em Problemas (ABP), na qual o acadêmico é instigado a obter o conhecimento a partir da discussão de casos-problema e através de uma busca ativa em fontes confiáveis de conteúdo. Além disso, por meio das atividades de Interação Ensino, Serviço e Comunidade (IESC), é possível atingir os objetivos propostos de uma educação integrada aos serviços do SUS, inserindo os alunos na rotina dos postos de saúde desde os primeiros anos de curso, sendo acompanhados por preceptores tanto médicos quanto profissionais de outras áreas da saúde envolvidos no atendimento de pacientes da comunidade ${ }^{2}$. 
No cenário de mudanças da medicina brasileira, destaca-se também a criação do Programa Mais Médicos, instituído inicialmente como medida provisória em 8 de julho de 2013 e aprovado na forma da lei $\mathrm{n}^{\mathrm{o}} 12.871$, de 22 de outubro de 2013. Em seu objetivo está o provimento de médicos para o atendimento da população usuária SUS ${ }^{3}$.

Para se cumprir esta meta, a abordagem prevê atuação em 3 frentes: 1- aumento da quantidade de vagas para a graduação de medicina, orientado pelas Diretrizes Curriculares Nacionais; 2aumento da carga horária do graduando em atividades relacionadas ao ensino na comunidade e, na pós-graduação, valorização dos Programas de Residência em Medicina Geral de Família e Comunidade; 3- Oferta de vagas para atendimento na atenção básica à profissionais brasileiros, estrangeiros com diploma revalidado e médicos formados no exterior (inclusive brasileiros) em regime de intercâmbio médico internacional ${ }^{3}$.

No Amapá, o programa mais médicos conta com 123 profissionais em atendimento nas unidades de atenção básica em todo o estado, os quais tem supervisão acadêmica gerenciada pela UNIFAP através de 2 tutores e 17 supervisores. Em 2015, o curso de medicina iniciou uma integração com as equipes de saúde família que possuem profissionais do programa mais médicos, de forma a possibilitar aos acadêmicos do quarto ano de graduação acompanhar as equipes de saúde dessas unidades como parte da programação prevista no IESC.

A iniciativa de realizar modificações na execução de atividades de ensino na comunidade no Amapá e permitir a discussão pelos próprios alunos envolvidos nesse processo incentivou o desenvolvimento desta pesquisa, a qual tem como objetivo identificar as percepções dos acadêmicos de medicina sobre a experiência de aprendizado na comunidade durante acompanhamento de equipes de saúde da família do programa mais médicos através da técnica do grupo focal.

\section{METODOLOGIA}

Trata-se de uma pesquisa de abordagem qualitativa para identificar as percepções de discentes sobre o aprendizado na comunidade durante acompanhamento de equipes de saúde da família integradas ao programa mais médicos para o Brasil. Estudo realizado no curso de Medicina da Universidade Federal do Amapá referente às atividades de ensino na comunidade do primeiro semestre da quarta série de graduação no ano letivo de 2015. A amostra foi formada por nove discentes do quarto ano de graduação do curso de medicina. Para inclusão no estudo, usou-se como critérios a assinatura de termo de consentimento livre e esclarecido e ser aluno regularmente matriculado na quarta série de graduação.

Para conhecer a percepção dos estudantes sobre o objeto de estudo, foi empregada a técnica baseada nos fundamentos teóricos do grupo focal. Por tratar-se de pesquisa qualitativa com base na análise de conteúdo do grupo estudado, não houve necessidade de cálculo de amostra, respeitandose apenas a recomendação de formar grupos de no mínimo seis e no máximo dez integrantes, em 
conformidade com os preceitos técnicos deste tipo de coleta de dados. Assim, foi realizada uma sessão de grupo focal com presença de nove alunos voluntários, na qual as questões disparadoras foram estruturadas para identificar os conhecimentos e percepções de discentes sobre a experiência de aprendizado nas atividades de ensino na comunidade conforme roteiro previamente elaborado para esta finalidade.

Grupo focal caracteriza-se por ser técnica de pesquisa que utiliza sessões grupais como um dos foros facilitadores da expressão de características psicossociológicas e culturais, cujos sujeitos do estudo discutem vários aspectos de um tópico específico. Os grupos focais configuram, assim, uma técnica de pesquisa que coleta dados por meio das interações grupais ao se discutir um tópico especial sugerido pelo pesquisador. Como técnica, ocupa uma posição intermediária entre a observação participante e as entrevistas em profundidade. Pode ser caracterizada também como um recurso para compreender o processo de construção das percepções, atitudes e representações sociais de grupos humanos ${ }^{4}$.

O roteiro temático abordado foi elaborado enfocando os itens que interessavam ser investigados dentro do tema de experiência de aprendizado de medicina na comunidade nas equipes do programa mais médicos. O grupo focal iniciou com a apresentação do moderador, do observador e dos participantes. Após os esclarecimentos sobre a finalidade da reunião e, após assinatura do termo de consentimento livre e esclarecido, solicitou-se permissão para gravação. Os objetivos da pesquisa foram explicados, e realizado discurso para motivar os sujeitos da pesquisa, assegurando-lhes que sua colaboração seria imprescindível para o êxito do trabalho. Houve garantia de anonimato da entrevista e sigilo da autoria das respostas.

O roteiro usado na sessão (Quadro 1) foi composto por 3 categorias de perguntas: perguntas de abertura ("O que o aluno achava do ensino na comunidade no quarto ano?/"Como o ensino na comunidade auxilia na formação médica?"), perguntas de exploração (“Como tem sido a experiência de participar das equipes de saúde da família do programa mais médicos?"/"O que a experiência tem proporcionado em sua formação?” e perguntas de finalização (“Existe mais alguma opinião a acrescentar sobre o tema de ensino na comunidade e programa mais médicos?”).

Os relatos apresentados no grupo focal foram gravados e transcritos posteriormente, buscandose evidenciar os temas emergentes da discussão entre os participantes. Os temas foram obtidos mediante a apreensão das unidades de significado presentes e reunidas no discurso dos sujeitos participantes do grupo, e analisados conforme sua pertinência em relação às dimensões da experiência vivenciada pelos estudantes na participação de equipes do programa mais médicos. $\mathrm{Na}$ transcrição, a gravação foi ouvida para conferência do discurso, e quando necessário, introduzidas alterações de pontuação do texto para facilitar a leitura ou a compreensão.

A análise dos conteúdos da entrevista do grupo focal foi realizada em duas etapas: inicialmente com a identificação dos temas mais citados nas falas dos participantes com utilização da ferramenta 
Wordle.net, a qual permite separar as palavras mais citadas durante as discussões; posteriormente, obedeceu-se a técnica de Bardin (2009) para análise de conteúdo ${ }^{5}$. Dessa forma, todo o material transcrito foi analisado e seus conteúdos divididos em categorias temáticas a partir das palavras identificadas inicialmente, depois houve agrupamento por frases e posteriormente por parágrafos para que as ideias fossem organizadas em categorias temáticas que permitissem inferências sobre os significados apresentados no grupo focal.

A presente pesquisa seguiu todos os princípios éticos da Declaração de Helsinque, sendo preservada a confidencialidade das fontes de informações. Todos os voluntários assinaram o Termo de Consentimento Livre e Esclarecido para participar deste estudo ${ }^{6}$. Além disso, foram respeitados todos os preceitos e orientações referentes a Ética em Pesquisa envolvendo Seres Humanos conforme dispositivos presentes na Resolução n ${ }^{\circ} 466$ de 12 de dezembro de 2012, a qual atualizou as diretrizes e normas regulamentadoras deste tema no país ${ }^{7}$. O projeto foi submetido ao Comitê de Ética em Pesquisa (CEP) da Universidade Federal do Amapá (UNIFAP), conforme procedimentos da Plataforma Brasil e aprovado sem restrições conforme Protocolo CAAE ${ }^{\circ}$ 48327615.4.0000.0003.

\section{RESULTADOS E DISCUSSÃO}

A análise de contéudo do grupo focal demonstrou cinco temas relacionados às percepções dos alunos sobre a experiência de aprendizado na comunidade durante o acompanhamento de equipes de saúde da família do programa mais médicos: PMMB e experiência na comunidade, integração com a medicina brasileira, níveis de atenção em saúde, rotina na atenção básica e relação médico paciente.

\section{PMMB e experiência na comunidade}

O Programa Mais Médicos surgiu em 2013 com uma proposta de formar recursos humanos na área médica para o SUS, diminuindo as desigualdades regionais e fortalecendo a atenção primária ${ }^{8}$. Entretanto, foi observado que sua implantação não foi capaz de alterar desigualdades internas, seja pelo contingente insuficiente para suprir as demandas atuais de médicos no sistema de saúde ou pelo fato de que estes acabaram alocados em localidades onde já existiam quantidade de médicos brasileiros dentro do mínimo recomendado pela Organização Mundial de Saúde (OMS) ${ }^{9}$.

Ao se analisar as respostas dos alunos que participaram do grupo focal, identificou-se que as opiniões gerais quanto ao programa divergem (Figura 2). Alguns apresentam a percepção de que se trata apenas de uma iniciativa política, como se pode verificar no enunciado:

"Acho, totalmente, que esse programa tem viés politico. Acho que é melhor do que nada, mas trata-se de uma resolução imediatista". 
Tal ideia vem sendo discutida por autores como Ribeiro (2015), que refere o programa como uma medida paliativa para aumento de contingente de médicos a curto e longo prazo, sem no entanto garantir estratégias para problemas estruturais e de formação de competência técnica para o sistema de saúde público ${ }^{10}$. Depreende-se então que a concepção deste grupo de alunos não é fato isolado e pode ser compreendida no contexto nacional, uma vez que essas observações também estão presentes na visão de outros autores. Contudo, tais afirmações não apresentam consenso, pois mesmo dentro da amostra de estudo, representada pelo grupo focal, pôde-se observar uma dicotomia no modo de interpretar esta realidade.

Quando se avaliam as respostas dos alunos que tem uma percepção do programa que foge ao contexto político, entende-se que em sua visão o programa apresenta um caráter social relevante, o que pode ser demonstrado na fala:

"O programa ajudou muitas famílias e suas realidades, que poderiam não ser mudadas por muitos anos por conta da carência de médicos no interior".

Sobre esse ponto de vista é importante lembrar que antes do Programa Mais Médicos outras estratégias não obtiveram sucesso em fornecer o atendimento médico necessário para populações mais isoladas ${ }^{8}$. Portanto, a forma encontrada pelo PMMB para ampliar a abrangência das ações em saúde pela alocação de profissionais estrangeiros em locais onde há deficiência de quantitativo médico é tida como uma proposta que tem trazido benefícios à população, auxiliando a atenção primária em seu objetivo principal que é levar atenção integral a toda a população.

A maior parte dos alunos entenderam a experiência com os médicos do programa como uma importanteforma de aprendizado, pois representa a oportunidade, sobretudo, de melhorar a relação médico-paciente, de poder se integrar a uma equipe de atenção primária e contribuir no próprio desenvolvimento pessoal. Tais ideias podem ser encontradas nas falas:

"Uma coisa que eu aprendi com os cubanos é que eles me fazem ser uma pessoa melhor".

"Às vezes estamos muito preocupados com detalhes técnicos do tratamento que a gente se esquece dessa humanização (...) acho que esse contato com os médicos do Programa Mais Médicos possibilita muito que melhoremos nesse sentido".

As atividades de inserção do aluno na comunidade se encontram em consonância com a proposta das novas DCN's, que passaram a dar maior ênfase para a integração dos acadêmicos na atenção primária em saúde e demais serviços do SUS com estímulo ao aumento na carga horária dos acadêmicos na atenção primária ${ }^{1}$. Para Santos et al. (2014), quando se deposita muita importância no conhecimento técnico em detrimento das habilidades práticas, há prejuízo no desenvolvimento do aluno por impedir o completo entendimento da complexidade de uma interação com pacientes reais ${ }^{11}$. 
O fato desta conexão entre ensino em sala de aula e ensino na comunidade ser bem avaliada pelos alunos e reconhecida como uma forma efetiva de aprimorar atitudes frente ao atendimento da população demonstra uma evolução das escolas médicas no caminho para a construção de um ensino mais humanizado. A inserção dos discentes nos ambientes de atendimento dos profissionais do PMMB como forma de implementar as ações de ensino em comunidade demonstrou uma avaliação positiva na percepção do grupo focal.

Outra questão identificada durante as discussões do grupo foi a forma como os alunos perceberam a utilização de protocolos de atendimento, na visão deles alguns dos médicos estrangeiros acompanhados pelos alunos deixaram de seguir as condutas preconizadas pelos principais consensos e diretrizes utilizados no Brasil, ressaltando a necessidade atualização e adaptação do conhecimento para a realidade brasileira, como exposto na seguinte fala:

"Minha crítica é que eles não estudam os protocolos daqui, não estudam artigos científicos, e assim não têm como apresentar pra gente discussão de terapêutica”.

O aspecto de educação permanente demonstra ter potencial para melhorar, segundo os dispositivos da Lei $n^{0} 12.871$, é obrigatório aos profissionais atuantes no PMMB a participação em atividades de aperfeiçoamento e educação permanente, sob orientação de tutores e supervisores ${ }^{3}$. A análise conjunta da crítica apresentada durante a discussão do grupo focal e do trecho citado da lei que rege o Programa Mais Médicos demonstra que a necessidade de um plano de educação continuada já foi observada no momento da implantação e, portanto, se faz de suma importância para que as medidas de ampliação do atendimento médico ocorram não somente em contingente de profissionais, mas também em qualidade de atendimento dos mesmos.

\section{Integração com a medicina brasileira}

Embora haja consenso entre os alunos participantes do grupo focal sobre a relevância de acompanhar os médicos do programa nos serviços de atenção primária, foi ressaltada a necessidade de maior integração destes profissionais com as atividades propostas pelo desenho curricular de medicina da UNIFAP. Esta ideia foi ressaltada nos seguintes trechos:

"Se eles estivessem inseridos no nosso curso, talvez ficasse mais fácil de raciocinarem do que estamos precisando".

\section{“(...) eles não sabem em que nível a gente está e acabam limitando nossas atividades”.}

Conhecer os diferentes cenários de aprendizado nas 3 esferas do SUS dão ao aluno maior entendimento sobre as políticas de saúde e o funcionamento das equipes multiprofissionais ${ }^{1}$. O entendimento dos alunos da UNIFAP sobre a relevância de fundamentar a prática da comunidade apresenta ressonância nas modificações do ensino médico nos últimos anos pelo inceitvo a inserção discente nas redes de serviço de saúde desde o início do curso. 
Esta realidade também pode ser observada no relato de Santos et al.(2014) ${ }^{11}$, o qual afirma ser essencial a integração entre a educação médica e o sistema de saúde, porém ressalta que esta parceria muitas vezes se dá de maneira opcional e heterogênea, tornando-a menos eficiente. Detecta-se então que o possível insucesso a ser corrigido reside na capacidade do curso em inserir naturalmente os profissionais do PMMB na realidade de ensino da universidade, fazendo com que tenham uma melhor percepção das necessidades de aprendizado e do conhecimento já adquirido. Tal medida pode ter desfecho eficaz em aprimorar a relação de confiança entre alunos e preceptores e permitir uma otimização da relação de aprendizado entre ambos.

Outro aspecto bastante citado durante a discussão foi a ideia preconcebida dos médicos brasileiros com relação aos profissionais do PMMB. De forma geral, toda a forma de preconceito foi desaprovada pelos alunos. Outros ainda admitiram ter mudado de opinião após contato com os médicos do programa, conforme evidenciado no trecho:

"No inicio fomos muito tendenciados a ter preconceito com os profissionais do programa e para mim foi uma experiência muito gratificante, para de certa forma, quebrar preconceitos que haviam dentro de mim, no sentido de julgar o trabalho de outrem sem conhecer".

"Com certeza a questão do preconceito e todos esses estigmas, pelo menos eu não tinha, mas ficava com aquele receio que todo mundo falava".

Em determinados serviços torna-se comum a difusão de idéias pré-concebidas à profissionais estrangeiros ou formados no exterior, muitas podem ser as dúvidas quanto à eficácia e qualidade do ensino de outros locais. ${ }^{12}$ Não se tem como precisar a gênese do preconceito muitas vezes expresso por outros profissionais em relação aos médicos estrangeiros, porém, apesar de uma publicidade inicial negativa, pôde-se observar que a interação mais próxima foi capaz de modificar a percepção de alguns alunos e desconstruir o receio inicial quanto à eficácia do atendimento realizado por profissionais do PMMB.

Um ponto favorável à experiência com médicos cubanos evidenciado pelos alunos, foi a disposição para o serviço e o ensino demonstrado pelos profissionais do programa (Figura 1). Em contraponto a isso, foi relatado que a própria equipe de saúde muitas vezes se opõe ao tempo gasto pelos médicos com os alunos, pois geralmente passam do horário previsto de atendimento ou simplesmente o cumprem a carga horária da forma integral. Pode-se observar estas ideias nas seguintes falas:

"Eles não estão de brincadeira, estão aqui para trabalhar e possuem um compromisso com a saúde do paciente."

"Mas o cubano era legal, dedicado e preocupado e extremamente irritado com a equipe, porque era o único que queria trabalhar" 
Observa-se que esta característica dos médicos atuantes no programa se encontra em conformidade com os princípios de interação entre ensino, serviço e comunidade preconizados tanto pelas diretrizes curriculares nacionais quanto pela Lei que rege o Programa Mais Médicos ${ }^{\mathbf{1}}$ 3. As ações dentro da comunidade se fazem como parte essencial do aprendizado para ambos, médicos e alunos, como forma de consolidar o conhecimento construído e aplicar o mesmo à população, além de garantir um ambiente de constantes trocas de saberes ${ }^{13}$.

\section{Níveis de atenção em saúde e Rotina na atenção básica}

O tópico atenção primária demonstrou boa percepção no que se refere a abordagem ao paciente como um todo e melhor integração das equipes de saúde com a comunidade atendida. Estes aspectos apresentam relevância em virtude de serem fundamentais ao desenho curricular delineado no projeto pedagógico do curso de medicina da UNIFAP $^{2}$, tornando o acompanhamento dos médicos do PMMB marcante para o aprendizado acadêmico. Estas ideias podem ser identificadas nas seguintes falas:

"Então eu acho que essa relação médico-paciente na comunidade é um treinamento bem melhor do que o que você vê em um atendimento mais complexo."

“(...) em um serviço de alta complexidade pode mudar o médico que está no plantão, pode mudar a pessoa que estar atendendo, enquanto que o médico que trabalha na atenção primária sempre vai estar ali."

A importância do aprendizado de relação médico-paciente em cenários de atuação profisional ficou mais evidente com a reestruturação do modelo de exercício da medicina, baseado em atender as necessidades da comunidade, assim como de aliar os ideais humanitários da profissão médica com a boa técnica. Para isto, é importante a participação e o engajamento das escolas médicas para a priorização do ensino na comunidade. Assim, este modelo deve estar sustentado na saúde e não na doença, com foco na comunidade e não no hospital, com médicos de visão abrangente e não especialistas, e que favoreçam a humanização e a prevenção na condução de seus pacientes ${ }^{14}$.

Foi ressaltado positivamente por grande parte dos participantes a capacidade dos médicos do programa em se integrar, com certa facilidade, à prática do atendimento primário em saúde do Brasil. Desse modo, o contato mais próximo com a realidade do paciente e a capacidade de poder acompanhá-lo como um todo, resultado dessa integração, também foram apontados como pontos positivos da experiência. Como pode ser observado nos seguintes trechos:

"O meu médico do semestre passado foi muito bom, por que ele estava muito inserido nesse contexto de saúde da família mesmo"

\section{“(...) mostrou ter uma noção muito grande de atendimento da atenção primária”}


“(...) na comunidade a gente tem essa oportunidade de visitar a casa”

"E na atenção primária a gente consegue observar esse início, esse meio e esse fim do contato que tu tens com o teu paciente."

A naturalidade de integração entre ensino da medicina e comunidade assistida pode ser alcançada com maior facilidade em anos iniciais do curso quando os acadêmicos são incluídos na comunidade e estimulados a adquirir experiências na medicina da família. Com esse tipo de estratégia, o discente passa a se habituar com as manifestações iniciais de doenças assim como acompanhar evolução dos quadros mais comuns dentro do território social de convívio com os pacientes. Outro ponto importante, desenvolvido na experiência na comunidade, corresponde à visão holística que o estudante adquire, buscando investigar não apenas o diagnóstico e tratamento adequado, mas também a avaliação de fatores ambientais como fonte de influência na evolução de uma doença. Esse aprendizado permite maior facilidade ao futuro profissional para atuar nos aspectos de prevenção e promoção de saúde ${ }^{15}$.

Os alunos especularam ainda sobre os motivos de muitos dos médicos brasileiros se negarem a trabalhar no interior ou em locais mais afastados da cidade, fazendo surgir a necessidade do PMMB preencher estas lacunas no atendimento. Foi discutido como a falta de melhores condições de trabalho e a falta de incentivo à ida dos profissionais para localidades afastadas dos grandes centros foram fatores condicionantes para o surgimento desta necessidade. Estas ideias estão apresentadas nas seguintes falas:

“(...) além de ter o grave problema da falta de estrutura para trabalhar, tem a questão da falta de incentivo"

“(...) essa realidade se trata da falta do estímulo ao médico brasileiro ir ao interior”

"O médico brasileiro, apesar de as vezes ter um bom salário, ele não vai no lugar onde o cubano está por causa das más condições da saúde brasileira."

A falta de condição de trabalho associado a precárias estruturas de assistência médica no interior do país pode ser muito bem evidenciada na região Norte, pois estudos sobre a distribuição de médicos na Amazônia, indicaram que existem muitas diferenças entre a quantidade de médicos disponíveis em cada região do Brasil. A Região Norte possui um quantitativo de médicos inferior ao restante do país, e na comparação da distribuição de profissionais nas capitais e nos interiores, a região Norte, junto com o Nordeste, possui pior distribuição, chegando a ter de 6 a 10 vezes mais médicos nas capitais. Ainda assim, a taxa de profissionais que trabalham no SUS chega a média de $65,5 \%$, com o acesso a médicos na saúde suplementar de 4,6 vezes maior que no SUS.

No Amapá, entretanto, $85,9 \%$ dos médicos trabalham no SUS e em alguns interiores da região norte esse percentual chega a $100 \%$, com a peculiaridade de muitos médicos trabalharem no interior, 
mas residirem na capital. Os dados ressaltam a necessidade de políticas para suporte e fixação de médicos nessa região, principalmente no interior, pois mesmo com salários atraentes, a falta de uma infraestrutura e condições de trabalho adequadas no interior, prejudicam a consolidação do profissional na localidade. Por isso, o papel do SUS é de essencial importância na assistência de saúde para a região Norte, e políticas como o Programa de Valorização da Atenção Básica (PROVAB) e o Programa Mais Médicos permitem elevar o provimento de médicos para a região Norte. Entretanto, há necessidade de estudos que permitam avaliar o real impacto dessas ações na resolução de problemas estruturais e na elevação da qualidade dos serviços de saúde na região ${ }^{16}$.

\section{Relação médico paciente}

Um ponto que o grupo entrou em consenso quanto aos médicos estrangeiros, foi sobre a valorização da relação médico-paciente. Em geral, os alunos concordaram que os médicos do programa apresentam boa relação com seus pacientes, pois passam confiança ao acompanhar a rotina da comunidade de perto. Informam ainda que acham que o acompanhamento a estes profissionais deveria começar no primeiro ano de graduação para estimular a humanização da educação médica e do cuidado. Tais percepções podem ser verificadas nos trechos:

“(...) detalhes que a gente às vezes não percebe, do tato com o paciente, de ser educado, de pedir licença. ",

\section{“(...) eles atendem muito bem, dão atenção para o paciente”}

"Eu acho que tinha que colocar o mais médicos desde o primeiro ano. Eu acho que facilitaria muito desde os primeiros anos essa questão de conversar com os pacientes."

A instituição do Programa Mais Médicos para o Brasil facilitou uma aproximação entre os acadêmicos de cursos de medicina e os profissionais envolvidos na execução das diretrizes do programa, foi possível criação de convênios e parcerias entre universidades, prefeituras e unidades de saúde. Assim, o ambiente de ensino pôde permitir trocas de experiências com maior significado, prioncipalmente em relação a forma humanística de abordagem ao paciente que procura a atenção básica ${ }^{8}$.

A percepção de aperfeiçoamento do contato entre médico e seu paciente pode auxiliar para o cumprimento dos objetivos do programa, mesmo com dificuldades estruturais, e melhorar o perfil da formação médica. O aprimoramento e auto-regulação do programa realizado pela sociedade e, no caso desta pesquisa, pela academia permitirão favorecer novas oportunidades de atividades em cenários de prática além de sugerir novos caminhos para o desenvolvimento de políticas de educação permanente e a promoção de troca de esperiências entre médicos brasileiros e de outros países ${ }^{8}$.

Nessa perspectiva, adotar de forma articulada uma prática médica cujo centro seja a comunidade, 
favorece a construção de vínculos entre discentes e pacientes, com maior confianca e participação de todos. Promover essa abordagem garante melhoria de aspectos de prevenção e promoção da saúde e, como desfecho de longo prazo, a redução de custos em todo o sistema de saúde ${ }^{17}$.

\section{CONSIDERAÇÕES FINAIS}

Este artigo buscou interpretar as experiências dos alunos de graduação de medicina da UNIFAP no contato com as equipes e profissionais participantes do programa mais médicos. Houve a constatação de aspectos positivos como a abordagem dos pacientes na comunidade, de forma holística e estabelecendo relação médico-paciente eficaz. A experiência de atendimento na comunidade, possibilitou redução de conceitos de desaprovação anterior que boa parte dos alunos tinham em relação aos profissionais do PMMB.

Temas relacionados à educação continuada e atualização foram demonstrados como aspectos negativos e que podem ser melhorados pela supervisão do programa, principalmente quanto ao melhor entendimento dos protocolos nacionais pelos médicos do programa.

A integração de ensino, serviços e comunidade com o PMMB no Amapá aumentou o entendimento dos alunos sobre a importâncias das práticas na comunidade e o potencial de aprendizado que esse eixo permite na graduação de medicina.

Além disso, os acadêmicos relataram como a falta de condições estruturais de muitas unidades básicas de saúde, principalmente aquelas situadas no interior, influenciam na qualidade do serviço oferecido à população. Fato evidenciado pela pouca motivação de médicos brasileiros em seguir, ou mesmo iniciar, carreira nos interiores do país e não apenas por questões salariais.

Em virtude da complexidade desse tema e do pouco tempo no qual o PMMB encontrase instituído, há necessidade de novos estudos que possam avaliar com maior profundidade o panorama das mudanças e experiências ocasionados por essa nova realidade. Identificar se padrões semelhantes de perspectivas são encontrados em outras regiões do país torna-se importante para maior reflexão sobre os modelos de políticas de saúde do país e sua interface com as universidades.

\section{REFERÊNCIAS BIBLIOGRÁFICAS}

1. Brasil. Ministério da Educação. Diretrizes Curriculares Nacionais do Curso de Graduação em Medicina. Brasília, DF: O Ministério; 2014.

2. Brasil. Universidade Federal do Amapá, UNIFAP. Pró-Reitoria de Ensino e Graduação. Projeto Pedagógico do Curso de Medicina. Macapá, 2013.

3. Brasil. Lei n ${ }^{\circ} 12.871$, de 22 de outubro de 2013. Instituição do Programa Mais Médicos. Diário Oficial da União 23 out 2013. 
4. Veiga L, Gondim SMG. A utilização de métodos qualitativos na ciência política e no marketing político. Opinião Pública. 2001; 7(1), 1-15..

5. Bardin L. Análise de conteúdo (Edição revista e actualizada). Lisboa: Edições, 70., 2009

6. Associação Médica Mundial. Declaração de Helsinque. Princípios éticos para a pesquisa em seres humanos. Helsinque, 1964.

7. Brasil. Conselho Nacional de Saúde.. Resolução nº 466, de 12 de dezembro de 2012. Diretrizes e normas regulamentadoras de pesquisa envolvendo seres humanos. Disponível em:http://conselho.saude.gov.br/resolucoes/2012/Reso466.pdf. Acesso em: 25 ago. 2015.

8. Oliveira FP, Vanni T, Pinto HA, Santos JTR, Figueiredo AM, Araújo SQ, Matos MFM. Mais Médicos: um programa brasileiro em uma perspectiva internacional. 2015

9. Brasil. Conselho Federal de Medicina, CFM., Conselho Regional de Medicina do Estado de São Paulo, CREMESP. Demografia Médica no Brasil. Cenários e indicadores de distribuição Vol 2. 2013.

10. Ribeiro RC. Programa Mais Médicos-um equívoco conceitual. Revista Ciência \& Saúde Coletiva. 2015; 20(2).

11. dos Santos FrancoI CAG, CubasI MR, FrancoI RS. Currículo de Medicina e as Competências Propostas pelas Diretrizes Curriculares. Revista Brasileira de Educação Médica. 2014; 38(2), 221-230.

12. dos Santos SEQUEIRA, D. H., \& LIMA, D. M. A. "Todo estrangeiro para brasileiro é cubano?": Identidade de médicas de cabo verde em cuba e no brasil.-DOI: 10.5216/teri. v4i1. 34004. Revista Terceiro Incluído. 2014; 4(1), 116-134.

13. dos Santos FrancoI CAG, CubasI MR, FrancoI RS. Currículo de Medicina e as Competências Propostas pelas Diretrizes Curriculares.Revista Brasileira de Educação Médica. 2014; 38(2), 221-230.

14. Troncon, LDEA. Ensino clínico na comunidade. Medicina (Ribeirao Preto. Online). 1999; $32(3)$.

15. Oliveira HL. Medical education and development. World Health Organization Regional Office for the Eastern Mediterraneum, EMRO/71/370, 1971.

16. Silveira RP, Pinheiro R. Entendendo a necessidade de médicos no interior da Amazônia- 
$95 / /$

Brasil. Rev. bras. educ. méd. 2014; 38(4), 451-459.

17. Fernandes, JCL. A quem interessa a relação médico-paciente. Cad Saúde Pública, 9(1), 21-27.1993.

Artigo apresentado em 25-09-15

Artigo aprovado em 05-12-15

Artigo publicado no sistema em 30-12-15 